\title{
news news news news news news news news
}

\section{SAJP GOES OPEN ACCESS}

The South African Journal of Psychiatry is moving to 'open access' through the open access online biomedical research publisher BioMed Central.

From the SAJP's perspective its publishing policy will remain essentially unchanged, with peer review continuing as before. There will also be citation tracking, with the addition of on-line tracking options (Scopus and Google Scholar), which will enable authors to monitor their own citation statistics directly. Copyright for articles is retained by authors, licensed under the Creative Commons Attribution License, which allows articles to be freely downloaded from the BioMed Central website and re-used and re-distributed without restriction, as long as the original work is correctly cited.

Open access will significantly broaden the reach of the SAJP, effectively making it global, and ensuring that the contents get the widest distribution.

Source: www.biomedcentral.com

\section{NEUROLOGICAL DISORDERS AND THE PUBLIC HEALTH CHALLENGES}

\begin{abstract}
Neurological disorders, including dementia, epilepsy, headache disorders, multiple sclerosis, neuroinfections, neurological disorders associated with malnutrition, pain associated with neurological disorders, Parkinson's disease, stroke and traumatic brain injuries, affect up to one billion people worldwide, according to a new survey by the World Health Organization ( $\mathrm{WHO}$ ).

Of these, some 50 million people suffer from epilepsy and 24 million from Alzheimer's or other dementias, and together these disorders, along with Parkinson's disease, are estimated to have accounted for $1.3 \%$ of total global DALYs in 2005 . This number is expected to rise to $1.5 \%$ in 2015 and more than $1.8 \%$ by 2030 - an increase brought about by a demographical transition from predominantly youthful populations to older and ageing ones as a result of increased life expectancy and reduced fertility.
\end{abstract}

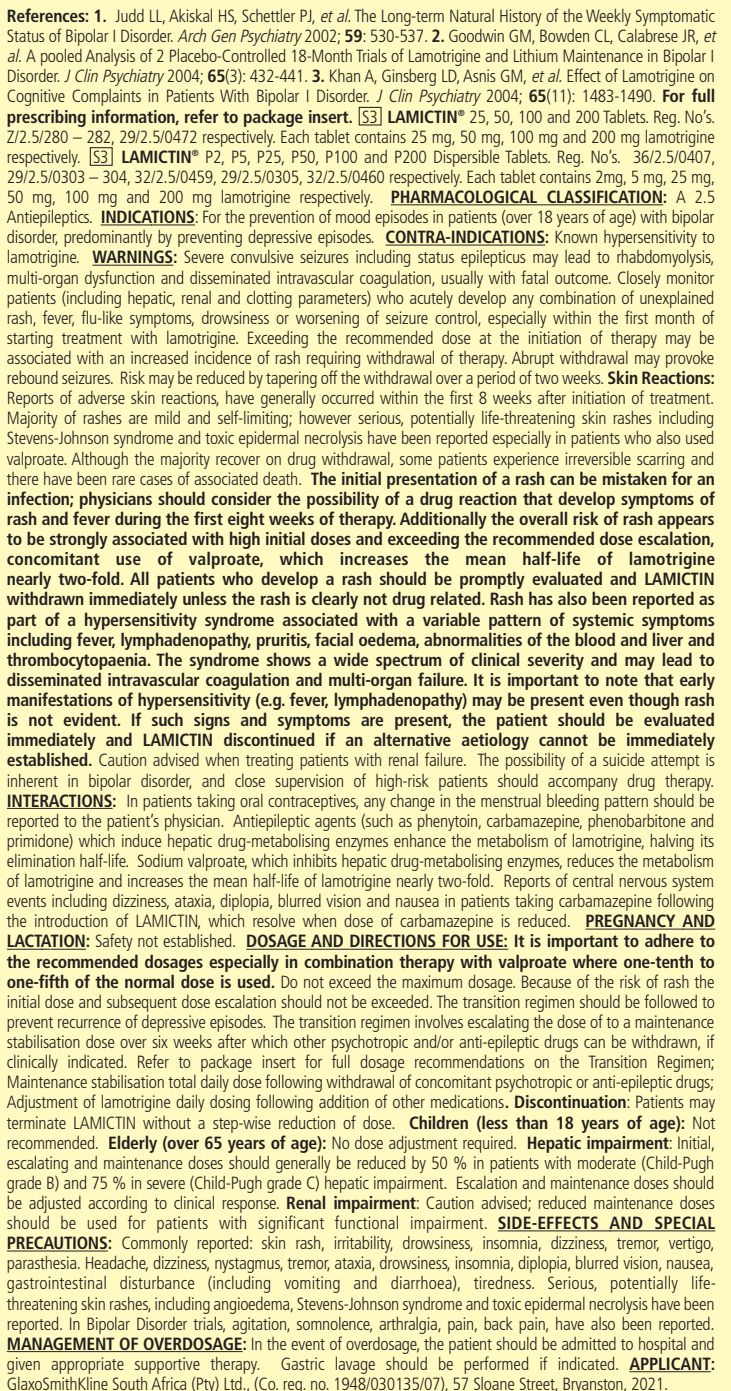

References: 1. Judd LL, Akiskal HS, Schettler PJ, et al. The Long-term Natural History of the Weekly Symptomatic
Status of Bipolar I Disorder. Arch Gen Psychiatry 2002; 59: 530-537. 2. Goodwin GM, Bowden CL, Calabrese JR, et

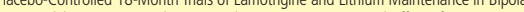
Cognitive Complints in Patients With Bipolar I Disorder I Clin Psychiatry 2004: 65 (11): 1483-1490. For full prescribing information refer to package insert. S33 LAMICTIN $25,50,100$ and 200 Tabots. Reg. No's. respectively 532 . $29 / 2.5 / 0472$ respectively. Each tablet contains $25 \mathrm{mg}, 50 \mathrm{mg}, 100 \mathrm{mg}$ and $200 \mathrm{mg}$ lamotrig's 29/2.5/0303 - 304, 32/2.5/0459, 29/2.5/0305, 32/2.5/0460 respectively. Each tablet contains $2 \mathrm{mg}, 5 \mathrm{mg}, 25 \mathrm{mg}$ Antiepileptics. INDICATIONS: For the prevention of mood episodes in patients (over 18 years of age) with bipol isorder, predominantly by preventing depressive episodes. CONTRA-INDICATIONS: Known hypersensitivity multi-organ dysfunction and disseminated intravascular coagulation, usually with fatal outcome. Closely monitor patients (including hepatic, renal and clotting parameters) who acutely develop any combination of unexplaine statting treatment with lamotrigine. Exceeding the recommended dose at the initiation of therapy may be ssociated with an increased incidence of rash requiring withdrawal of therapy. Abrupt withdrawal may provole Reports of adverse skin reactions, have generally occurred within the first 8 weeks atter initiation of treatment Stevens-Johnson syndrome and toxic epidermal necrolysis have been reported especially in patients who also used
valproate. Athough the majority recover on drug withdrawal, some patients experience irreversible scarring and there have been rare cases of associated death. The initial presentation of a rash can be mistaken for oxe strongly associated with high initial doses and exceeding the recommended dose escalation,

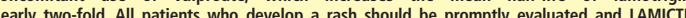
part of a hypersensitivity ss th

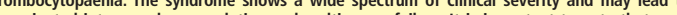
manifestations of hypersensitivity (eg fever lymphadenopathy) may be present even though ras

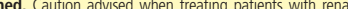
NTERACTIONS: In patients taking oral contraceptives, any change in the menstrual bleeding pattern should primidone) which induce hepatic drug-metabolising enzymes enhance the metabolism of lamotrigine, halving its elimination half-life. Sodium valproate, which inhibits hepatic drug-metabolising enzymes, reduces the metabolis events including dizziness, ataxia, diplopia, blurred vision and nausea in patients taking carbamazepine following ACTATION: Safety not Est

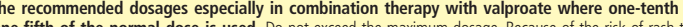

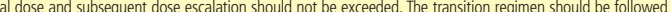
stabilisation dose over six weeks after which other psychotropic and/or anti-epileptic drugs can be withdrawn, clinically indicated. Refer to package insert for full dosage recommendations on the Transition Regimen: Adjustment of lamotrigine daily dosing following addition of other medications. Discontinuation: Patients may (less than 18 years of age): Not scalating and maintenance doses should generally be reduced by $50 \%$ in patients with moderate (Child-Pug (rade B) and $75 \%$ in severe (Child-Pugh grade Cl hepatic impairment. Escalation and maintenance doses should should be used for patient wh sigificant functional impairment. SIDE-EFFECTS AND SPECI prasthesia. Headache, dizziness, nystagmus, tremor, ataxia, drowsiness, insomnia, diplopia, blurred vision, nausea,

\section{Depicting a new standard for bipolar disorder dominated by depression'}

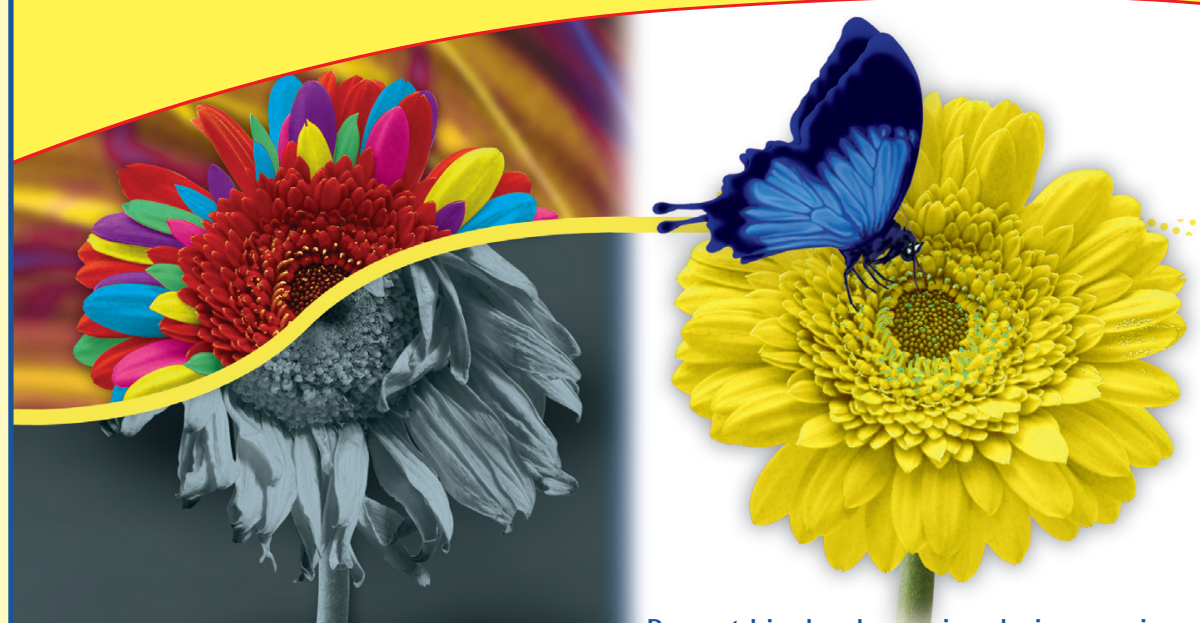

Prevent bipolar depression during manic or depressive episodes ${ }^{2}$

without destabilising mood $^{2}$

whilst improving cognitive functioning ${ }^{3}$
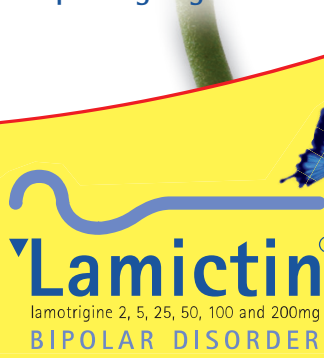

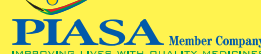




\section{Providing quality of life to those caught in the grip of neuropsychological illness}

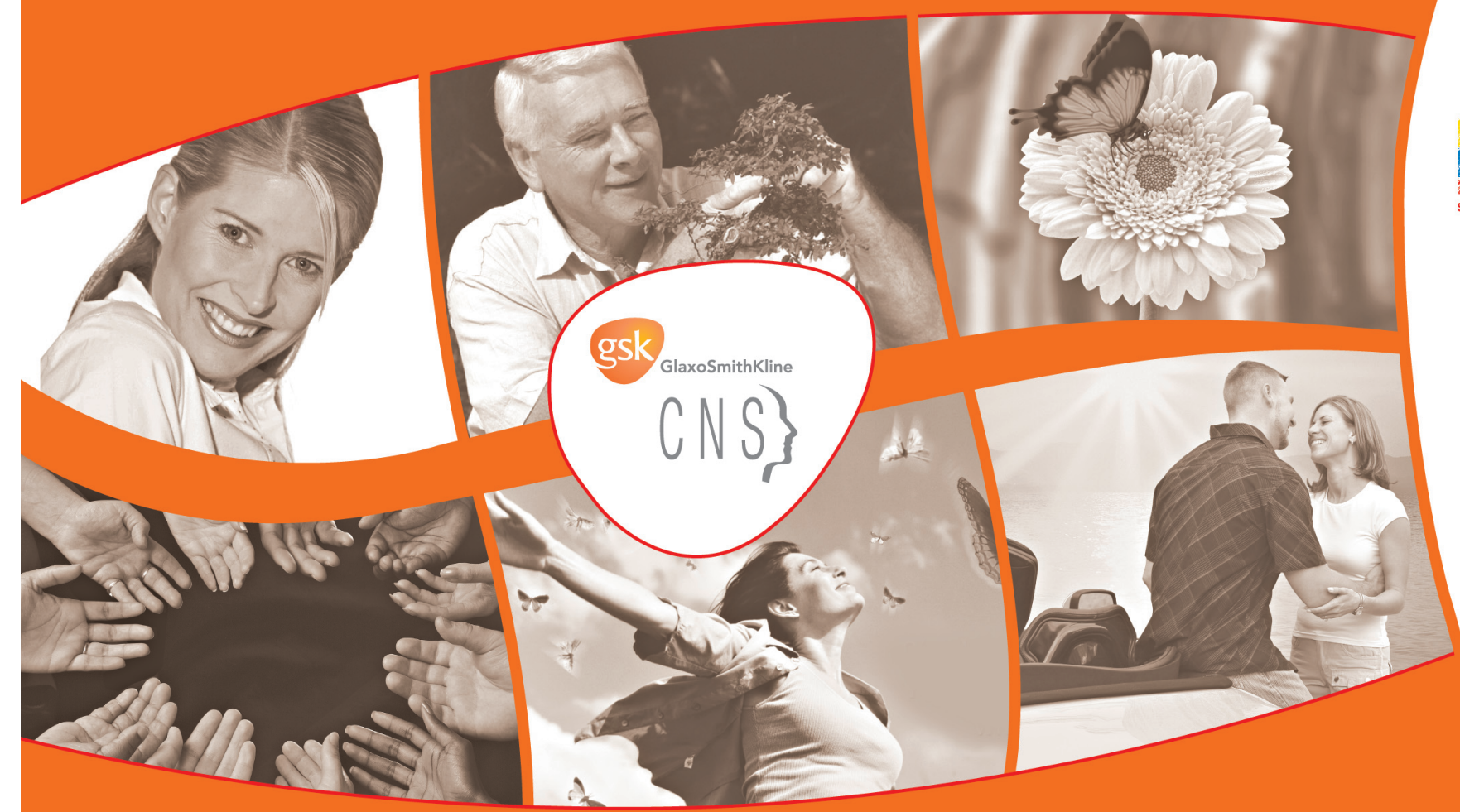

These disorders are found among all age groups and in all geographical regions.

Despite the fact that effective, low-cost treatments are available, large numbers of people go untreated for reasons including inadequate health delivery systems, lack of trained personnel, the absence of essential drugs and the prevalence of traditional beliefs and practices.

The report says that a clear message emerges: unless immediate action is taken globally, the neurological burden is expected to become an even more serious and unmanageable threat to public health. Accordingly it recommends a series of 'simple but effective' actions, including greater commitment from decision makers, increased social and professional awareness, strategies that address stigma and discrimination, national capacity building and international collaboration. The research agenda for developing countries, including operational research, also needs to be developed to gain better understanding of the problem so that appropriate responses can be developed and evaluated.

Most importantly, neurological care may be integrated into primary health care. In these settings, doctors can use lowtechnology interventions, and community-based rehabilitation is also an option.

Source: www.who.int

\section{GlaxoSmithKline launches HIV/AIDS and Mental Health Newsletter}

Neuropsychiatric disorders comprise the second largest component of HIV/AIDS, our national burden of disease.

The National Research Foundation has provided funding for a cross-university brain-behaviour initiative (CUBBI), a research initiative focused on the psychobiology of vulnerability and resilience after psychological trauma.

GSK initiated this newsletter, in association with the CUBBI, as a service to the medical community The newsletter will provide practitioners with a series of updates on HIV/AIDS and mental health, written by respected professionals in their individual fields of expertise. It also aims to raise awareness and highlight the role and gravity of mental health in HIV/ AIDS patients

Professor Dan Stein, Professor and Chair of the Department of Psychiatry, University of Cape Town and Director, MRC Unit on Anxiety and Stress Disorders, University of Stellenbosch, has been appointed as editor. The first issue appeared at the end of June 2007.

Parties interested in receiving or contributing to future issues of the newsletter are welcome to contact Madelein Steyl, CNS Brand Manager, GSK South Africa, tel 1011 1) 745-6046 or e-mail: madelein.m.steyl@gsk.com.

Reference available on request 